PANORAMA

ISSN: $1909-7433$

ISSN: 2145-308X

ednorman@poligran.edu.co

Politécnico Grancolombiano

Colombia

\title{
DESARROLLO DE LAS COMPETENCIAS CIENTÍFICAS MEDIANTE LA IMPLEMENTACIÓN DEL APRENDIZAJE BASADO EN PROBLEMAS (ABP) EN LOS ESTUDIANTES DE GRADO QUINTO DEL INSTITUTO UNIVERSITARIO DE CALDAS (MANIZALES)
}

Duque Cardona, Valentina; Largo-Taborda, Wilson Alejandro

DESARROLLO DE LAS COMPETENCIAS CIENTÍFICAS MEDIANTE LA IMPLEMENTACIÓN DEL

APRENDIZAJE BASADO EN PROBLEMAS (ABP) EN LOS ESTUDIANTES DE GRADO QUINTO DEL INSTITUTO UNIVERSITARIO DE CALDAS (MANIZALES)

PANORAMA, vol. 15, núm. 28, 2021

Politécnico Grancolombiano, Colombia

Disponible en: https://www.redalyc.org/articulo.oa? $\mathrm{id}=343965146008$

Institución Universitaria Politécnico Grancolombiano

Institución Universitaria Politécnico Grancolombiano

\section{(c) $(1) \Theta$}

Esta obra está bajo una Licencia Creative Commons Atribución-NoComercial-SinDerivar 4.0 Internacional. 
Artículos de investigación científica y tecnológica

PANORAMA, vol. 15, núm. 28, 2021

Politécnico Grancolombiano, Colombia

Recepción: 05 Septiembre 2020 Aprobación: 21 Noviembre 2020

Redalyc: https://www.redalyc.org/ articulo.oa?id=343965146008
DESARROLLO DE LAS COMPETENCIAS CIENTÍFICAS MEDIANTE LA IMPLEMENTACIÓN DEL APRENDIZAJE BASADO EN PROBLEMAS (ABP) EN LOS ESTUDIANTES DE GRADO QUINTO DEL INSTITUTO UNIVERSITARIO DE CALDAS (MANIZALES)

DEVELOPMENT OF SCIENTIFIC COMPETENCES THROUGH THE IMPLEMENTATION OF PROBLEMBASED LEARNING (PBL) IN FIFTH GRADE STUDENTS OF THE INSTITUTO UNIVERSITARIO DE CALDAS (MANIZALES)

DESENVOLVIMENTO DE COMPETÊNCIAS CIENTÍFICAS ATRAVÉS DA IMPLEMENTAÇÃO DA APRENDIZAGEM POR PROBLEMAS (PBL) NOS ALUNOS DO QUINTO GRAU DO INSTITUTO UNIVERSITARIO DE CALDAS (MANIZALES)

Valentina Duque Cardona valentina.duque@ucm.edu.co Universidad Católica de Manizales., Colombia Wilson Alejandro Largo-Taborda wlargo@ucm.edu.co Universidad Católica de Manizales., Colombia

(D) https://orcid.org/0000-0002-4718-8763
Resumen: El Aprendizaje Basado en Problemas (ABP) es una estrategia metodológica que presenta al docente y al estudiante un grupo de actividades que buscan que el aprendizaje sea activo, dinámico y significativo; esta estrategia tiene como punto de partida situaciones problémicas que surgen de la cotidianidad del estudiante, logrando así un aprendizaje con sentido. Para el desarrollo de la investigación se tuvo como objetivo la implementación del ABP para el desarrollo de las competencias científicas para los estudiantes de grado quinto en la enseñanza del sistema respiratorio. La metodología implementada se dio desde el enfoque cualitativo, método investigaciónacción, con un grupo experimental y un grupo control, en el Instituto Universitario de Caldas, de la ciudad de Manizales. Para la recolección de la información se tuvo en cuenta el nivel de apropiación que tienen los estudiantes sobre las competencias científicas, para luego llevar a cabo la implementación del $\mathrm{ABP}$ en la enseñanza de la temática relacionada con el sistema respiratorio y así evaluar el desarrollo de las competencias científicas en los estudiantes de dicho grado. A modo de conclusión, los estudiantes de grado quinto del 
instituto Universitario de Caldas presentaron un avance en la apropiación y desarrollo de las competencias científicas, siendo un proceso más consciente y significativo, logrando un aprendizaje desde la experiencia y las propias vivencias.

Palabras clave: ABP, competencias científicas, estrategia pedagógica, innovación educativa, unidad didáctica.

Abstract: Problem-Based Learning (PBL) is a methodological strategy that presents the teacher and the student with a group of activities that seek to make learning active, dynamic and meaningful; This strategy is based on problem situations that arise from the student's daily life, thus achieving meaningful learning. For the development of the research, the objective was to implement the ABP for the development of scientific competencies for fifth grade students in teaching the respiratory system. The implemented methodology was given from the qualitative approach, research-action method, with an experimental group and a control group, in the Instituto Universitario de Caldas, in the city of Manizales. For the collection of the information, the level of appropriation that the students have on the scientific competences was taken into account, to then carry out the implementation of the PBL in the teaching of the subject related to the respiratory system and thus evaluate the development of the scientific competences in students of said degree. By way of conclusion, the fifth grade students of the Instituto Universitario de Caldas presented an advance in the appropriation and development of scientific competences, being a more conscious and meaningful process, achieving learning from experience and their own experiences.

Keywords: PBL, Scientific competencies, Pedagogical strategy, Educational innovation, Teaching unit.

Resumo: A Aprendizagem Baseada em Problemas (PBL) é uma estratégia metodológica que apresenta ao professor e ao aluno um conjunto de atividades que buscam tornar a aprendizagem ativa, dinâmica e significativa; Essa estratégia é baseada em situaçõesproblema que surgem no dia a dia do aluno, alcançando assim uma aprendizagem significativa. Para o desenvolvimento da pesquisa, objetivou-se implementar o PAF para o desenvolvimento de competências científicas para alunos do quinto ano no ensino do aparelho respiratório. A metodologia implementada deu-se a partir da abordagem qualitativa, método pesquisa-ação, com grupo experimental e grupo controle, no Instituto Universitario de Caldas, na cidade de Manizales. Para a coleta das informações, foi levado em consideração o nível de apropriação que os alunos possuem sobre as competências científicas, para então realizar a implantação do PBL no ensino da disciplina relacionada ao sistema respiratório e assim avaliar o desenvolvimento de as competências científicas nos alunos do referido grau. A título de conclusão, os alunos do $5^{\circ}$ ano do Instituto Universitario de Caldas apresentaram um avanço na apropriação e desenvolvimento das competências científicas, sendo um processo mais consciente e significativo, conseguindo aprender com a experiência e as próprias vivências.

Palavras-chave: ABP, habilidades científicas, estratégia pedagógica, inovação educacional, unidade didática.

\section{INTRODUCCIÓN}

Esta investigación surge de la necesidad de encontrar estrategias para llevar al aula de clase las temáticas propias del grado quinto en el área de Ciencias naturales, de manera que permitiera generar un cambio en la dinámica del aula y una transformación que fortalecería las competencias científicas evaluadas por el Ministerio de Educación Nacional (MEN), por medio de la prueba saber $5^{\circ}$ (Díaz, 2006; Escobedo, 2001).

Es allí donde el $\mathrm{ABP}$ surge como una alternativa para solucionar dicho problema y romper el paradigma tan arraigado de la educación tradicional que prevalece en esta institución (García, 1999; Guerrero, 2019); el ABP permite relacionar la cotidianidad del estudiante con los conceptos que 
son propios de cada grado de escolaridad, en este caso particular, el grado quinto (Gil \& De Guzmán, 1993).

Sastre y Araujo (2018), explican que el ABP se posiciona como un enfoque innovador en los procesos formativos y académicos que se llevan a cabo en la actualidad. Su importancia se visualiza desde la educación básica, media y secundaria; en esta última toma cada vez mayor auge, es decir, se convierte en una posibilidad para que los docentes puedan relacionar los conceptos con la experiencia y la práctica (Camacho, Casilla \& Finol, 2008).

En concordancia con lo anterior, Betancourt (2006) y Pérez (2015) indican la importancia del ABP para el desarrollo de la autonomía de los estudiantes, ya que se focaliza en los estudiantes y no en el profesor. Igualmente, busca que sean ellos los encargados de su aprendizaje, puesto que el rol de estos es activo y permanente. Por ende, la motivación de los estudiantes aumenta por convertirse en parte de la solución de problemas que se plantean relacionados con la realidad y el contexto (Giraldo, Zuluaga \& Naranjo, 2020).

En perspectiva de lo anterior, el proyecto se implementó en el Instituto Universitario de Caldas con los estudiantes de grado quinto; dichos estudiantes oscilan entre los 9 y 12 años, se contó con una muestra total de 81 alumnos divididos en dos grupos, 5A Y 5B, por ello, se determinó tener un grupo experimental y un grupo control respectivamente, para así comparar los resultados de ambos grupos en un pretest a manera de diagnóstico, y un postest después de implementar la estrategia en el grupo experimental (López, Marulanda \& Piedrahita, 2011; Martínez, 2011).

La estrategia ABP fue implementada por medio de una unidad didáctica basada en la temática del sistema respiratorio; se eligió esta porque permite reunir en su estructura los ocho pasos propios del ABP, según Morales y Landa (2004). Un resultado importante mostró que la estrategia del $\mathrm{ABP}$ afectó positivamente el desarrollo de las competencias científicas, además, la capacidad analítica y la interpretación de textos de toda índole, lo cual impacta directamente la motivación, la curiosidad y la posibilidad de construir y desarrollar un conjunto de habilidades básicas cognitivas, procedimentales y de actitud para permitir que los estudiantes se conviertan en actores que puedan aprender con sentido (Gutiérrez et al., 2018; Marín, Parra, Burgos \& Gutiérrez, 2019).

En ese sentido, el ABP permite que los estudiantes puedan tener un rol activo y critico dentro de su proceso formativo, ya que son ellos los encargados de proponer soluciones reales a problemas reales. Por este motivo, el ABP permite el desarrollo de las competencias científicas: el uso comprensivo del conocimiento científico, la indagación y la explicación de fenómenos mediante la implementación de un proceso guiado, organizado e intencionado, lo cual permite vincular el concepto con una problemática que debe ser solucionada por el estudiante (Calderón, 2012). 


\section{UNIDAD DIDÁCTICA}

Según Moreira (2010), la unidad didáctica permite realizar una evaluación constante de los procesos que van de la mano del trabajo del estudiante, es decir, la planeación, la evaluación y la ejecución de las estrategias usadas por los docentes; además, permitió incluir en su estructura los ocho pasos propuestos por Morales y Landa (2004) para la ejecución del $\mathrm{ABP}$ en estudiantes de Básica primaria. Dichos pasos implican la lectura y el análisis del problema, una lluvia de ideas por parte de los participantes, una construcción de un listado de aquello que se conoce y se desconoce, adicionalmente se debe pensar en la problemática y en aquello que se requiere para solucionarlo, por tal motivo es indispensable definir el problema, obtener, analizar y presentar los resultados.

Para iniciar la presente investigación se llevó a cabo una revisión de fuentes bibliográficas que aportaron insumos para enriquecer el proceso que se llevaría a cabo y tener bases sólidas y concretas para obtener resultados fiables. La aplicación del pretest y el postest se realizó mediante Google forms, lo cual permitió tener unos resultados estadísticos e inmediatos del desempeño de los estudiantes antes y después de la implementación del ABP (Fuente, Manzanares \& Manzanares, 2006).

Las preguntas para estos dos cuestionarios se seleccionaron teniendo en cuenta las sugerencias realizadas por Alfarado (2019), autor que brinda elementos para categorizar las preguntas según el nivel de la competencia a evaluar.

\section{METODOLOGÍA}

Para el desarrollo del proyecto se trabajó mediante el tipo de investigación acción educativa (IAE), ya que permite seguir tres pasos fundamentales que van de la mano con los objetivos específicos de la presente investigación: planificar, implementar y evaluar; se tuvo en cuenta el enfoque cualitativo, porque con él se logró describir situaciones, acciones y estrategias observadas en el aula de clase, además, se logró estudiar el contexto y buscar solución al problema planteado (Elliot, 1991).

Por otro lado, se trabajó desde un enfoque cualitativo. Hernández, Fernández y Baptista (2010), lo conciben como un escenario en el cual las prácticas interpretativas permiten la comprensión del mundo, ya que lo hace visible, lo cambia y lo transforma en un panorama tangible o real, es decir, lo modifica a anotaciones, grabaciones, observaciones y documentos. Por tal motivo, investigador y participantes dialogan entorno a un problema que se desea abordar, modificar o evaluar, en este caso particular, se da en un entorno educativo (Álvarez, 2003; Cuenya \& Ruetti, 2010).

Los participantes se seleccionaron por conveniencia, debido a la cercanía que tenía con los estudiantes de grado quinto como docente de ciencias naturales del grupo experimental (41 estudiantes); el grupo control (40 estudiantes), contaba con otro docente, el cual usaba 
estrategias tradicionales de enseñanza. Los grupos son homogéneos en cuanto a estrategias metodológicas aplicadas, grupos de edades, situación socioeconómica, plantel educativo y nivel alcanzado de las competencias científicas antes mencionadas (Arteta et al., 2002; Coronado \& Arteta, 2015). Es importante recalcar que los estudiantes de grado quinto se encuentran en un momento académico que implica cambios, ya que continuarán con su avance en los niveles escolares de la educación secundaria.

Para darle respuesta a la pregunta problematizadora, se utilizaron tres instrumentos: inicialmente se llevó a cabo un proceso de identificación usando el pretest, que constó de nueve preguntas, cada pregunta se categorizó por competencia científica a evaluar y sus tres grados de dificultad según el ICFES en su prueba saber $5^{\circ}$; se implementó la unidad didáctica como mecanismo de intervención dentro del grupo experimental y al final se evaluó la intervención el postest, considerando el mismo mecanismo que el pretest.

\begin{tabular}{|c|c|c|}
\hline COMPETENCIA & NIVELES & $\begin{array}{c}\text { CANTIDAD DE } \\
\text { PREGUNTAS }\end{array}$ \\
\hline INDAGACIÓN & $\begin{array}{c}\text { Mínimo } \\
\text { Satisfactorio } \\
\text { Avanzado } \\
\text { USO COMPRENSIVO DEL } \\
\text { CONOCIMIENTO CIENTÍFICO }\end{array}$ & $\begin{array}{c}\text { Mínimo } \\
\text { Satisfactorio } \\
\text { Avanzado }\end{array}$ \\
\hline EXPLICACIÓN DE FENÓMENOS & $\begin{array}{c}\text { Míno } \\
\text { Satisfactorio } \\
\text { Avanzado }\end{array}$ \\
\hline
\end{tabular}

Tabla 1.

Estructura del pre-test y post-test Fuente: elaboración propia (2020).

\section{RESULTADOS}

Para realizar el análisis de la información, generar resultados y realizar recomendaciones se usó la prueba T-Student disponible en Excel, la cual generó comparaciones estadísticas entre el grupo control y el grupo experimental en cada una de las pruebas aplicadas, arrojando como resultado:

\begin{tabular}{|l|l|l|}
\hline & Variable 1 & Variable 2 \\
\hline Media & 16,11111 & 16,11111 \\
\hline Desviación & 15,61111 & 18,61111 \\
\hline Error típico de la media & 0,677 & 0,688 \\
\hline
\end{tabular}

Tabla 2.

Análisis de los resultados usando T-Student.

Fuente: elaboración propia (2020).

Como se muestra en la siguiente Gráfico, el pretest demostró que el grupo control y experimental son homogéneos, lo que indica que no muestran diferencias significativas en cuanto al nivel alcanzado de las 
competencias científicas por los estudiantes, que se encuentran en un nivel mínimo.

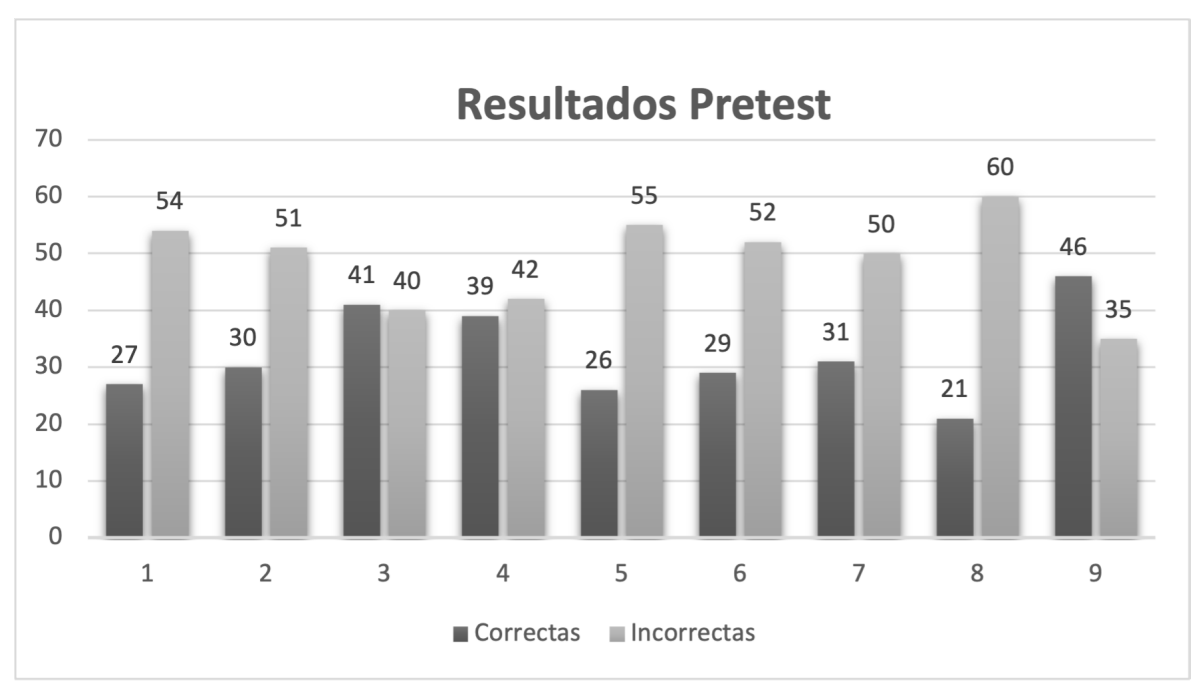

\section{Gráfico 1.}

Resultados de la prueba diagnóstica.

Fuente: elaboración propia (2020)

$\mathrm{Al}$ aplicar la unidad didáctica en los estudiantes del grupo experimental se observó apertura, entusiasmo y dinamismo en el aula, los estudiantes fueron actores activos del proceso de enseñanza y aprendizaje, se pudo observar con exactitud cada uno de los pasos del ABP y cómo les brindan a los estudiantes los elementos necesarios para darle respuesta a la situación problémica planteada.

$\mathrm{Al}$ aplicar el postest a ambos grupos se determinó que después de implementar la unidad didáctica el grupo experimental mostró avances significativos en la apropiación de los contenidos, aplicándolos en situaciones problema, dando así un nivel más alto en las competencias científicas. 


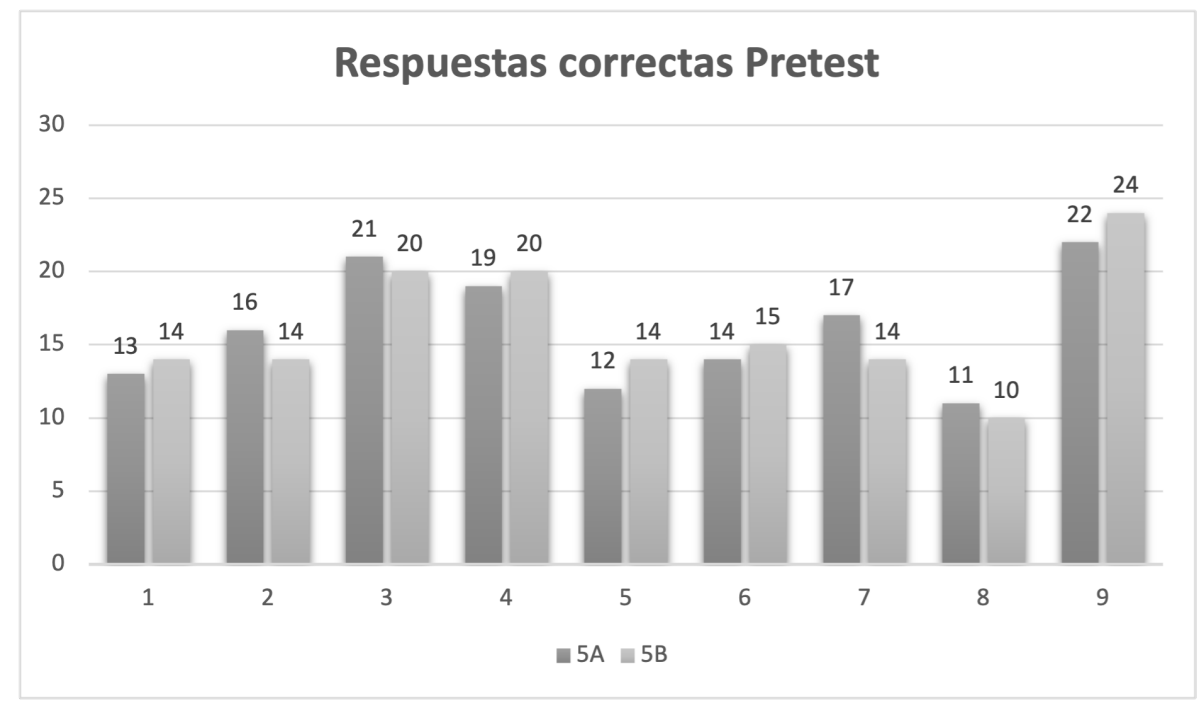

Gráfico 2.

Respuestas correctas postest grupo control vs grupo experimental.

Fuente: elaboración propia (2020).

Teniendo en cuenta la anterior gráfica se puede apreciar un avance importante reflejado en una diferencia entre las medias del pretest al postest. Esto se resume como una variación positiva para el grupo experimental en cuanto a las competencias científicas, que estaría asociada con implementación de la estrategia ABP, la cual se refiere a la importancia del trabajo colaborativo y la investigación, reflejándose en una mayor confianza en los conocimientos y conceptos que presentan los estudiantes y el uso de habilidades como la selección, la búsqueda, la organización y el análisis de la información a la que tienen acceso.

Igualmente, es importante considerar que los procesos que se adelantaron hasta la implementación del ABP fueron simultáneos en ambos grupos, es decir, se trabajó de manera tradicional con la clase magistral, pero de manera virtual a causa de la contingencia generada por la pandemia del Covid-19. 


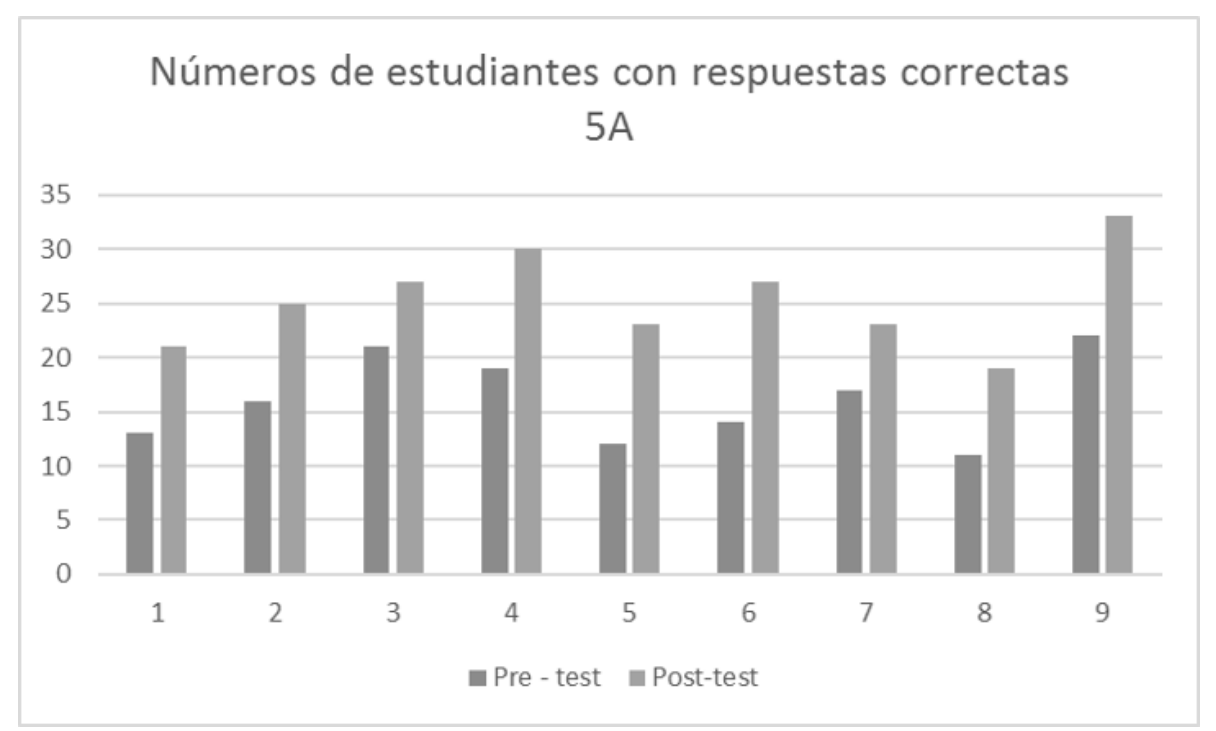

\section{Gráfico 3.}

Respuestas correctas de los estudiantes del grupo experimental pretest vs. postest. Fuente: elaboración propia (2020).

En la Gráfico 3 se puede verificar el avance que se presentó entre la implementación de los instrumentos del pre y el postest una vez terminada la intervención mediante la estrategia del ABP, el cual permitió un trabajo más consciente frente al desarrollo de las competencias, en este caso particular, las competencias científicas para ciencias naturales. Con base en lo planteado, se reconoce el avance que se dio entre el grupo control y experimental una vez terminada la intervención de la metodología de ABP, tal como se puede observar en la Gráfico 4.

En concordancia con lo anterior, el ABP permitió a los estudiantes un trabajo guiado, organizado y pertinente, ya que abordó el tema del sistema respiratorio desde un enfoque contextualizado donde los estudiantes se convierten en sujetos activos de su proceso formativo y académico.

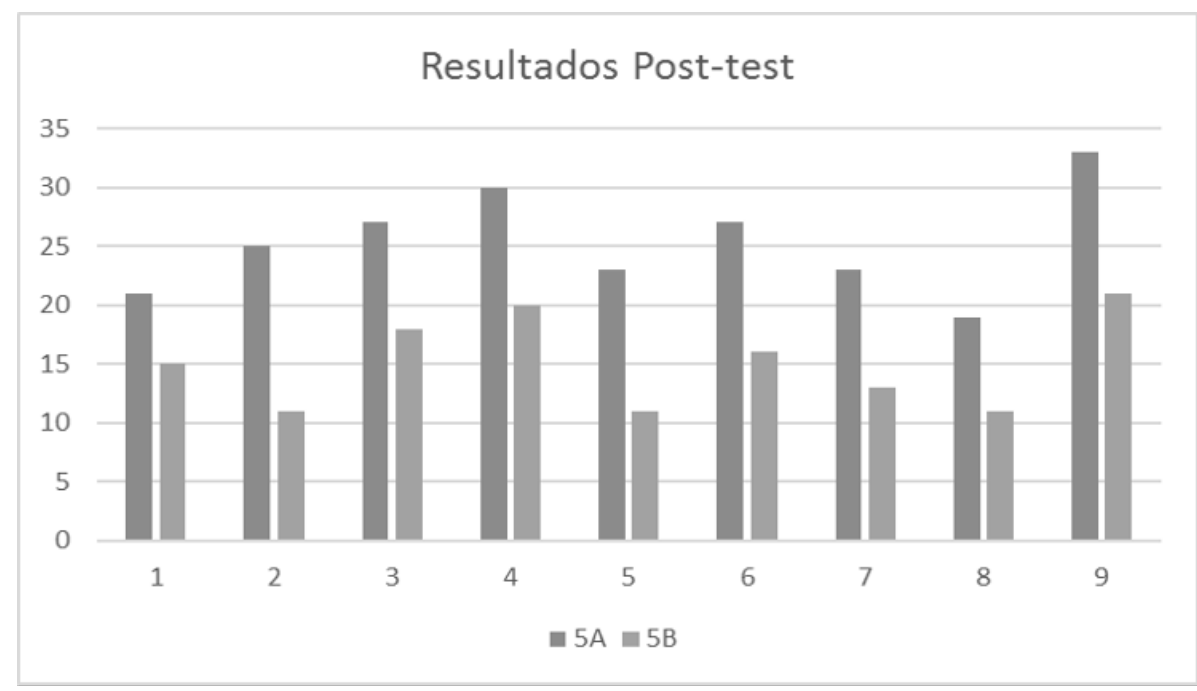

Gráfico 4.

Respuestas correctas postest grupo control vs grupo experimental. Fuente: elaboración propia (2020). 
Por último, el análisis del pretest y el postest para el grupo control puso en manifiesto que no hubo un cambio en el proceso relacionado con las competencias científicas, lo cual se puede analizar en la Gráfico 5.

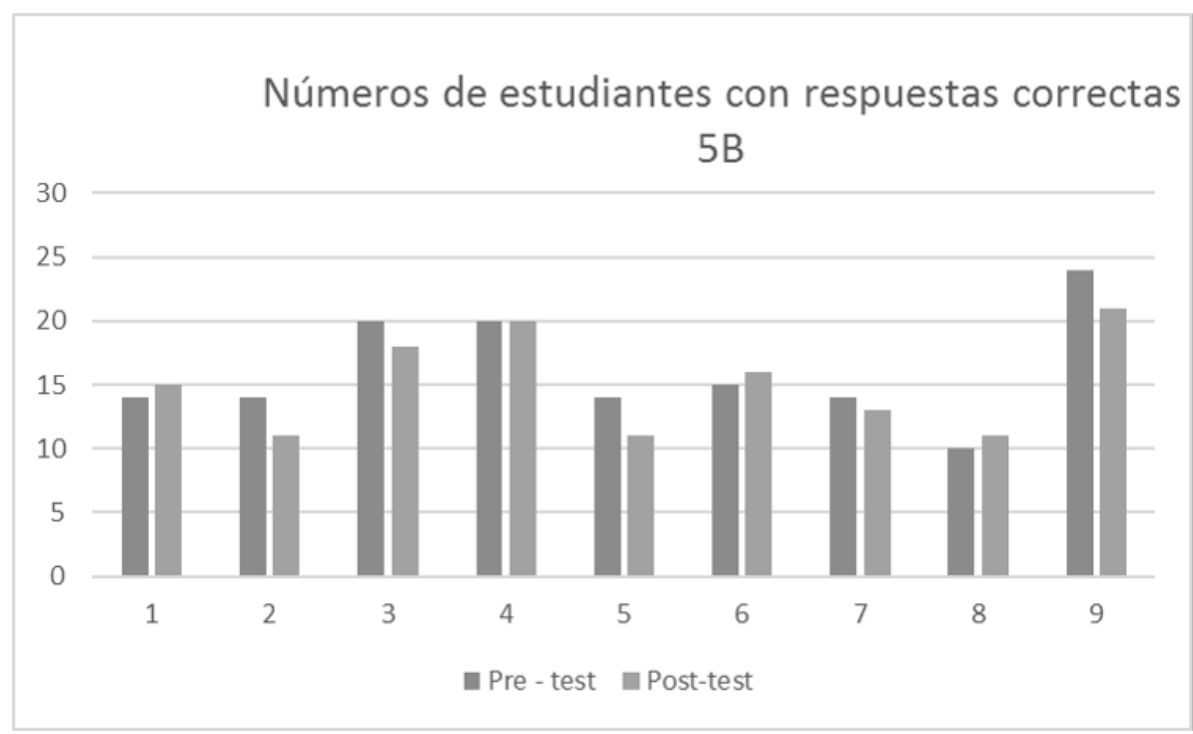

Gráfico 5 .

Respuestas correctas pretest y postest grupo control. Fuente: elaboración propia (2020).

\section{DISCUSIÓN Y CONCLUSIONES}

La aplicación de pruebas como el pretest y postest brinda información relevante para intervenir un grupo social, y poder analizarlos de una manera adecuada, es decir, permitió reconocer e identificar las necesidades de los estudiantes y el nivel que tenían a la hora de abordar la temática propuesta en conjunto con la implementación del $\mathrm{ABP}$.

El ABP brinda a los estudiantes mayores posibilidades para resolver problemas, utilizar sus conocimientos en contexto y desarrollar habilidades autodidactas, ya que desde la misma metodología se pretende una conexión entre el contexto y los problemas que se plantean para que los estudiantes se conviertan en sujetos activos del proceso formativo.

El ABP se puede usar como una estrategia para transformar la educación tradicional y darle un papel más activo al estudiante dentro de su propio proceso formativo, puesto que el $\mathrm{ABP}$ se convierte en un proceso innovador que va más allá de la enseñanza magistral y en el cual los estudiantes abordan problemas reales y que generan soluciones desde el conocimiento previo que ellos tienen.

Resulta fundamental contextualizar las clases con la realidad del estudiante, no solo para darle un aprendizaje con sentido, sino para despertar su interés y curiosidad. Este factor es trascendental para que el estudiante pueda avanzar en su proceso formativo, ya que la motivación hace parte intrínseca del aprendizaje y la enseñanza, puesto que si el estudiante se siente motivado podrá articular sus ideas con el aprendizaje contextualizado. 
Esta estrategia permite fortalecer las competencias científicas de los estudiantes, siguiendo con rigurosidad los ocho pasos propuestos para el ABP, y además se puede desarrollar otras habilidades interpretativas, propositivas, metacognitivas y un sinfín de habilidades que permiten además la nuclearización del currículo.

Es importante implementar la propuesta en el grupo control para que se pueda validar y reconocer la eficacia de la metodología del ABP para el desarrollo de las competencias científicas, con el fin de darle a ambos grupos la oportunidad de potenciar las competencias científicas y así mejorar dicho proceso en el grado quinto, con atención a continuar su proceso académico en la secundaria.

\section{REFERENCIAS}

Alfarado, V. (2019). Pautas en la Elaboración de Preguntas tipo ICFES-MEN. Seminario. http://acreditacion.unillanos.edu.co/CapDocentes/contenid os/7_jornada_pedagogica/pautas_elaborar_items_o_preguntas_tipo_icf es.

Álvarez-Gayou, J. (2003). ¿Cómo hacer investigación cualitativa? Fundamentos y metodología. Ecuador: Paidós.

Arteta, J., Chona, G., Perlaza, M., Fonseca, G., Martínez, S., \& Ibáñez, S. (2002). Las competencias científicas y el pensamiento de los profesores de ciencias naturales. El oficio de investigar. Educación y pedagogía frente a nuevos retos, 3. Colección Desarrollos en Investigación. https://doi.org/10.17227/ted.n um20-1061.

Betancourt, C. (2006). Aprendizaje basado en problemas una experiencia novedosa en la enseñanza de la ingeniería. Revista Educación en Ingeniería, I(2), 45-51. https://www.acofi.edu.co/revista/Revista2/archivpdf/2006 _II_13.

Calderón, Y. (2012). La formación de actitud científica desde la clase de Ciencias Naturales. Amazonia Investiga, 1(1), 36-53. https://doi.org/10.34069/A I/2012.01.02.3.

Camacho, H., Casilla, D., \& Finol, M. (2008). La indagación: una estrategia innovadora para el aprendizaje de procesos de investigación. Laurus, 14(26),284-306. https://www.redalyc.org/articulo.oa?id=761/7611149 1014.

Constitución Política de Colombia. (1991). Preámbulo. Bogotá, Colombia.

Coronado, M., \& Arteta, J. (2015). Competencias científicas que propician docentes de Ciencias naturales. Zona próxima, (23), 131-144.

Cuenya, L., \& Ruetti, E. (2010). Controversias epistemológicas y metodológicas entre el paradigma cualitativo y cuantitativo en psicología. Revista colombiana de Psicología, 19(2), 271-277. https://revistas.unal.edu.co/in dex.php/psicologia/article/view/17795.

Díaz, Á. (2006). El enfoque de competencias en la educación: ¿Una alternativa o un disfraz de cambio? Perfiles educativos, 28(111),7-36. http://www.sci elo.org.mx/scielo.php?pid=S0185-26982006000100002.

Elliot, J. (1991). Action research for educational change. United Kingdom: McGraw-Hill Education. 
Escobedo, H. (2001). Desarrollo de competencias básicas para pensar cientificamente. Una propuesta didáctica para Ciencias Naturales. Bogotá: Colciencias.

Fuente, A., Manzanares, M. C., \& Manzanares, M. (2006). Diseño y desarrollo de una unidad didáctica sobre desarrollo sostenible en maestros de educación primaria. Res Novae Cordubenses: estudios de calidad e innovación de la Universidad de Córdoba, (4), 227-257. https://dialnet.un irioja.es $/$ servlet $/$ articulo?codigo $=5227249$.

García, N. (1999). El aprendizaje basado en problemas (ABP): una estrategia didáctica para la indagación y la integración del conocimiento. La Gaceta Didáctica, 1, 9-11.

Gil, D., \& De Guzmán, M. (1993). Enseñanza de las Ciencias y las Matemáticas, tendencias e innovaciones. Organización de Estados Iberoamericanos (OEI).

Giraldo-Gómez, O., Zuluaga-Giraldo, J., \& Naranjo-Gómez, D. (2020). La proyección social, una apuesta desde el acompañamiento pedagógico. Praxis, 16(1). https://doi.org/10.21676/23897856.3033.

Guerrero, L. (2019). Aprendizaje Basado en Problemas (ABP) como estrategia para fortalecer las competencias científicas en ciencias naturales. PaideiaSurcolombiana, (24), 67-76. https://doi.org/10.25054/01240307 .1700 .

Gutiérrez, M., Gil, H., Zapata, M., Parra, L., \& Cardona, C. (2018). Uso de las herramientas digitales en la enseñanza y el aprendizaje universitario. Centro Editorial Universidad Católica de Manizales (UCM). http://hdl .handle.net/10839/2481.

Hernández, C. (2005). ¿Qué son las competencias cientificas? Foro Educativo Nacional, 1-30.

Hernández, R., Fernández, C., \& Baptista, P. (2010). Metodología de la investigación. México: McGraw-Hill.

Ley No 115. (1994). Ley General de Educación. Congreso de la República de Colombia, Bogotá, Colombia. http://www.mineducacion.gov.co/1621/a rticles-85906_archivo_pdf.

López, V., Marulanda, L., y Piedrahita, K. (2011). Concepciones sobre la digestión bumana en los niños y niñas de cuarto grado de primaria (Tesis de estudio, Universidad Tecnológica de Pereira). Repositorio Institucional UTP. htt ps://core.ac.uk/download/pdf/71396608.

Lorduy, O. (2014). Diseño de una propuesta didáctica utilizando el ABP como estrategia de enseñanza de la circulación sanguinea en el ser humano, en estudiantes de grado sexto. (Tesis de pregrado, Universidad Nacional de Manizales) Repositorio Institucional UNAL. https://repositorio.unal.ed u.co/handle/unal/53353.

Marín-Cano, M., Parra-Bernal, L., Burgos-Laitón, S. y Gutiérrez-Giraldo, M. (2019). La práctica reflexiva del profesor y la relación con el desarrollo profesional en el contexto de la educación superior. Revista Latinoamericana de Estudios Educativos, 15(1), 154-175. http://latinoam ericana.ucaldas.edu.co/downloads/Latinoamericana15(1)_9.

Martínez, J. (2011). Propuesta didáctica para la enseñanza del concepto de masa en los estudiantes del décimo grado de la Institución Educativa Raices delfuturo. (Tesis de maestría, Universidad Nacional de Colombia) Repositorio Institucional UNAL. https://repositorio.unal.edu.co/handle/unal/8351. 
Merriam, S. (2009). Qualitative research: A guide to design and implementation (2nd ed.) San Francisco, CA: Jossey-Bass.

Ministerio de Educación Nacional. (2006). Estándares Básicos de Competencias en Lenguaje, Matemáticas, Ciencias y Ciudadanas. Bogotá: Ministerio de Educación Educativa.

Montero, I., \& León, O. (2002). Clasificación y descripción de las metodologías de investigación en Psicología. International journal of clinical and health psychology, 2(3), 503-508. https://www.redalyc.org/pdf/337/33720308.

Morales, P., \& Landa, V. (2004). Aprendizaje basado en problemas. Theoria, 13. 145-157.http://biblioteca.udgvirtual.udg.mx/jspui/handle/123456789/ 574.

Moreira, M. (2011). Unidades de enseñanza potencialmente significativasUEPS Potentially Meaningful Teaching Units-PMTU. Aprendizagem Significativa em Revista/Meaningful Learning Review, 1(2), 43-63. http:/ /www.if.ufrgs.br/asr/artigos/Artigo_ID10/v1_n2_a2011.

Murillo, J., Gómez, N., \& Mejía, L. (2012). El desarrollo de competencias cientificas: una propuesta que integra el museo de la universidad de Antioquia como recurso didáctico, en la metodología del aprendizaje basado en problemas. (Tesis de pregrado, Universidad de Antioquia) Repositorio Institucional UdeA. http://hdl.handle.net/123456789/242.

Osorio, C. (2015). Aplicación de la metodología pequeños cientificos en las instituciones oficiales Manizales, Caldas, Occidente. Proyecto de inversión Alcaldía de Manizales 2015.

Páramo, D. (2015). La teoría fundamentada (Grounded Theory), metodología cualitativa de investigación científica. Pensamiento \& gestión, (39), 1-7. http://www.scielo.org.co/scielo.php?pid=S1657-62762015000200 001\&script $=$ sci_arttext\&tlng=pt.

Pérez, M. (2015). El ABP- Una estrategia didáctica en el desarrollo de proceso de pensamiento cientifico. Caso estudiantes séptimo grado de una institución educativa Floridablanca-Santander (Tesis de maestría, Universidad Industrial de Santander) Repositorio Institucional Tangara. http://tangara.uis.edu.co/biblioweb/tesis/2014/151755.

Restrepo, B. (2005). Aprendizaje basado en problemas (ABP): una innovación didáctica para la enseñanza universitaria. Educación y Educadores, 8, 9-19. https://www.redalyc.org/articulo.oa?id=834/83400803.

Ritter, J., \& García, A. (2012). Contribución a la adquisición de competencias científicas desde el aprendizaje basado en problemas. En: Congreso Internacional de Innovación Docente Universitaria en Historia Natural ( $1^{\circ}$. 2012. Sevilla), 182-188. Bioscripts. http://hdl.handle.net/11441/38730.

Sandín, M. (2003). Investigación cualitativa en educación. Fundamentos y tradiciones. Madrid: Mc Graw and Hill Interamericana.

Santillán, F. (2006). El Aprendizaje Basado en Problemas como propuesta educativa para las disciplinas económicas y sociales apoyadas en el BLearning. Revista iberoamericana de educación, 40(2), 3. https://www.me digraphic.com/cgi-bin/new/resumen.cgi?IDARTICULO=31634.

Servicio de Innovación Educativa. (2008). Aprendizaje Basado en Problemas. Madrid: Universidad Politécnica.

Sastre, G., \& Araujo, U. (2018). El aprendizaje basado en problemas (1ra ed). Editorial Gedisa. 
Tobón, S. (2013). Los proyectos formativos: transversalidad y desarrollo de competencias para la sociedad del conocimiento. Seminario. Universidad Complutense de Madrid. https://seminariorepensarlabioquimica.files.wo rdpress.com/2016/01/s26-srbq-fad910_sergio_tobon-_3_.

Universidad Politécnica de Madrid. (2008). Aprendizaje basado en problemas: guias rápidas sobre nuevas metodologias. Servicio de innovación educativa.

Vincent, T. (2014) Crafting Questions That Drive Projects. Strategies for Developing Powerful Inquiry Questions. https://learninginhand.com/blog /drivingquestions. 